OPEN ACCESS

Edited by:

Fei Mao,

Jiangsu University, China

Reviewed by:

Feng Ge,

Chinese Academy of Sciences, China

Yiqing Tian,

Xinyi People's Hospital, China

*Correspondence:

Pan Zhaoj

1169479680@qq.com

Niu Guoping

xz70707@163.com

Bian Maohong

mhbian@126.com

${ }^{\text {t}}$ These authors have contributed equally to this work

Specialty section: This article was submitted to Molecular and Cellular Oncology, a section of the journal

Frontiers in Oncology

Received: 19 May 2020 Accepted: 30 December 2020 Published: 10 February 2021

Citation:

Jian F, Yangyang $R$, Wei X, Jiadan $X$, Na L, Peng Y, Maohong B, Guoping N and Zhaoji $P$ (2021) The Prognostic and Predictive Significance of circRNA

CDR1as in Tumor Progression.

Front. Oncol. 10:549982. doi: 10.3389/fonc.2020.549982

\section{The Prognostic and Predictive Significance of circRNA CDR1as in Tumor Progression}

\author{
Fang Jian ${ }^{1 \dagger}$, Ren Yangyang ${ }^{2 \dagger}, \mathrm{Xu} \mathrm{Wei}^{1+}, \mathrm{Xu} \mathrm{Jiadan}^{3+}{ }^{+} \mathrm{Li} \mathrm{Na}^{1}$, Yang Peng ${ }^{1}$, \\ Bian Maohong ${ }^{1 *}$, Niu Guoping ${ }^{4 *}$ and Pan Zhaoji ${ }^{4 *}$ \\ ${ }^{1}$ Department of Blood Transfusion, The First Affiliated Hospital of Anhui Medical University, Hefei, China, ${ }^{2}$ Clinical \\ Laboratory, Xinyi People's Hospital, Xuzhou, China, ${ }^{3}$ Department of Clinical Medicine, The First Affiliated Hospital of Anhui \\ Medical University, Hefei, China, ${ }^{4}$ Clinical Laboratory, Xuzhou Central Hospital, The Affiliated XuZhou Hospital of Medical \\ College of Southeast University, Xuzhou, China
}

Cerebellar degeneration-related protein 1 antisense (CDR1as) is an important member of the circRNAs family, also known as cirs-7. Its main function in vivo is to act as a mir-7 sponge. Accumulated studies show that CDR1as is closely related to various diseases, especially cancer. Our analysis show that CDR1as expression in human cancer is significantly associated with poor overall survival (hazard ratio $[\mathrm{HR}]=2.50,95 \%$ confidence interval $[\mathrm{Cl}]=2.06-3.04 ; p<0.00001$ ) and that high CDR1as expression is associated with the tumor node metastasis stage (odds ratio $[\mathrm{OR}]=2.13,95 \% \mathrm{Cl}=1.63$ 2.78; $p<0.00001)$, and distant metastasis (OR $=3.50,95 \% \mathrm{Cl}=1.90-6.64 ; p<$ 0.00001). Furthermore, the results reveal the prognostic significance of CDR1as in neoplasms of the digestive system $(\mathrm{HR}=1.69,95 \% \mathrm{Cl}=2.14-2.71 ; p<0.001)$, colorectal cancer $(\mathrm{HR}=1.34,95 \% \mathrm{Cl}=1.96-2.85 ; p<0.001)$, and non-small cell lung cancer $(\mathrm{HR}=2.40,95 \% \mathrm{Cl}=3.42-4.83 ; p=0.008)$. In this study, we summarize in detail the latest research findings and demonstrate the function and regulatory mechanism of CDR1as in various cancer processes, and its potential as a biomarker for cancer prevention and prognosis.

Keywords: cerebellar degeneration-related protein 1 antisense, cancer, function, prognostic, predictive significance

\section{INTRODUCTION}

In recent years, cancer has become the most serious threat to human health. There were more than 1.68 million new cancer patients in the United States in 2017, and more than 600,000 people died of cancer (1). Therefore, the prevention and treatment of cancer is one of the most urgent problems. Circular RNAs (circRNAs) are a class of RNA molecules that lack the 5'-3' terminal and are covalently bound to form a closed loop and single-stranded transcripts from exons, introns, or intergenic regions $(2,3)$.

CircRNAs were first found in viroids in 1976, and were later isolated from several eukaryotes in 1979; they have been erroneously considered as transcriptional noise $(4,5)$. The role of circRNAs in disease regulation has not been adequately appreciated. However, with the rapid development of bioinformatics and high-throughput sequencing technology, the biological characteristics of 
circRNAs have been gradually revealed. Discovered in recent years, circRNAs are a large class of ncRNAs, widely found in a variety of eukaryotes, that participate in a variety of biological processes (6-8). Numerous studies have revealed the importance of circRNAs in regulating gene expression at both the transcriptional and post-transcriptional level. In addition, dyregulation of circRNA is regulated with the abnormal progression in various dieases, including cancer (9-14). To date, a variety of circRNAs have been shown to play a crucial role in human cancer initiation and development, including liver, lung, colorectal, breast, and prostate cancer (15). Sang et al. detected that circRNA_0025202 regulates tumor progression in breast cancer by regulating the $m i R-182-5 p / F O X O 3 a$ axis (16). CircHIPK3 is significantly upregulated in colorectal cancer (CRC) tissues and cell lines and is an independent prognostic factor of poor overall survival (OS). Knockdown of circHIPK3 markedly inhibited CRC cell proliferation, migration, invasion, and induced apoptosis in vitro and suppressed CRC growth and metastasis in vivo (17). Liu et al. implied that bladder cancer patients with high circ0001361 expression levels have a poor OS and that circ 0001361 promotes bladder cancer cell invasion and metastasis both in vitro and in vivo (18). Hsa_circ_0067934 is upregulated in esophageal squamous cell carcinoma (ESCC) tissues and may be a potential prognostic marker for ESCC (19). The upregulation of circRNA_100338 and downregulation of circMTO1, circ-ITCH, and cSMARCA5 are positively correlated with the poor prognosis of hepatocellular carcinoma (HCC) (20-23). It has been reported that Hsa_circ_0001649 has potential diagnostic and prognostic value for gastrointestinal tumors and may be a sensitive indicator for distant metastasis of gastric cancer and liver cancer (24-27). Thus, it is becoming increasingly clear that circRNAs are important in cancer pathogenesis. CircRNAs play important roles in the diagnosis, growth, metastasis, and treatment of drug resistance in cancer. Therefore, circRNAs are expected to be the new targets for tumor diagnosis and treatment $(28,29)$.

\section{MATERIALS AND METHODS}

\section{Literature Search Strategies}

The public databases PubMed, EMBASE, Web of Science, Wiley Online Library, and Medline were searched with the terms "CDR1NAT" or "ciRS-7" or "CDRlas" and "Cancer" or "Tumor" or "Neoplasia" and "prognos"” or "surviv" or "outcome" or "predict." The last search was on December 1, 2020.

\section{Inclusion and Exclusion Criteria}

All eligible study data were evaluated and extracted by two researchers; the relationship between CDRlas, tumor tissue, and patient survival was analyzed only when expression levels were reported. The inclusion criteria were as follows: (a) expression levels of CDR1as in cancer tissues; (b) correlation between the expression levels of CDR1as and survival outcomes (OS, progression-free survival [PFS], metastasis-free survival $[\mathrm{MFS}]$, or disease-free survival $[\mathrm{DFS}])$; (c) hazard ratio (HR) and 95\% confidence interval (CI) for survival time were reported or could be calculated from the reported data. Exclusion criteria were as follows: (a) Animal studies, case reports, meta-analysis articles, and reviews; (b) Papers lacking raw data or from which we could not calculate the $\mathrm{HR}, 95 \% \mathrm{CI}$, and $p$ values.

\section{Data Extraction}

We extracted the following data: first author's name, publication year, study region, sample size, cancer type, tumor size, tumor node metastasis (TNM) stage, the method of CDR1as testing, outcome, HR with 95\% CI, and HR statistics from each eligible study.

\section{Statistical Analysis}

STATA 12.0 (StataCorp, College Station, TX, USA) was used in this study. HR, OR, and corresponding 95\% CIs were used as indicators to evaluate each clinical parameter and patient prognosis or survival. $p<0.05$ was considered statistically significant.

\section{RESULTS}

\section{Biogenesis of CDR1as}

Cerebellar degeneration-related protein 1 antisense RNA (CDRlas), also known as asciRS-7, is a circRNA that is transcribed in the antisense orientation with respect to the CDR1 gene $(30,31)$. CDR1as is predominantly found in the human brain, it is 1485bp in length and located on the $\mathrm{X}$ chromosome (chrX: 139865339-139866824) (32). It functions as a miR-7 sponge/inhibitor in the embryonic zebra fish (33), and it is also expressed in a variety of tissues and organs in the human body. Its expression imbalance is associated with a variety of diseases, such as Alzheimer's disease, diabetes, myocardial infarction, and cancer (34-36). CDR1as has diverse biological functions and it is mainly involved in the proliferation, migration, and invasion of tumor cells. It regulates the biological function of tumors in two main ways (1): binding to the miRNA as a competing endogenous RNA through the base complementary pairing principle (2); regulating gene expression at the transcriptional and post-transcriptional level $(2,13,15$, 37). Numerous studies have shown that CDR1as is dysregulated in many human malignancies, such as CRC, non-small cell lung cancer (NSCLC), HCC, and gastric cancer (GC); they may serve as new diagnostic biomarkers and cancer treatment targets (38, 39). We systematically reviewed the literature to provide information on CDR1as expression patterns and roles in various tumors.

\section{The Multifaceted Role of CDR1as in Human Tumors CDR1as in Hepatocellular Carcinoma and Cholangiocarcinoma}

Recently, four research groups have analyzed the expression of CDR1as in HCC and its relationship with prognosis (40-43) (Table 1). Although the expression of CDR1as in HCC tissues remains controversial, upregulation of CDR1as is an independent risk factor for hepatic microvascular invasion 
TABLE 1 | Summary of the role of CDR1as in cancers so far.

\begin{tabular}{|c|c|c|c|c|}
\hline Cancer type & Function & $\begin{array}{l}\text { Expression } \\
\text { level }\end{array}$ & Intersection molecules and/or pathway & References \\
\hline NSCLC & Promoting cell proliferation, migration and invasion & Up & $\begin{array}{l}\text { CDR1as-miR-7/NF-kB or CDR1as-miR-7-EGFR/ } \\
\text { CCNE1/PI3K pathway; CDR1as-miR-219a-5p/SOX5 } \\
\text { pathway }\end{array}$ & $(44-47)$ \\
\hline $\mathrm{HCC}$ & Promoting hepatocellular microvascular invasion & Up & $\begin{array}{l}\text { CDR1as-miR-7-EGFR or PI3K/AKT/mTOR pathway; } \\
\text { CDR1as-miR-7-EGFR/CCNE1/PI3K pathway; } \\
\text { CDR1as-miR-7-KLF4 pathway }\end{array}$ & $(40-43)$ \\
\hline $\mathrm{CRC}$ & $\begin{array}{l}\text { Promoting cell proliferation, invasion and correlates with advanced } \\
\text { tumor stage, tumor depth and metastasis }\end{array}$ & Up & $\begin{array}{l}\text { CDR1as-miR-7-EGFR/IGF-1R; CDR1as-miR-7-EGFR/ } \\
\text { RAF1/MAPK pathway }\end{array}$ & $(50,51)$ \\
\hline ESCC & Promoting cell proliferation, migration and invasion & Up & $\begin{array}{l}\text { CDR1as-miR-7-HOXB13/NF-kB; CDR1as-miR-876- } \\
\text { 5p-MAGE-A family pathway; CDR1as-miR-7/KLF4/ } \\
\text { NF-kB pathway }\end{array}$ & $(52-54)$ \\
\hline Brain tumor & Inhibiting cell proliferation and migration & Down & $\begin{array}{l}\text { CDR1as-miR-671-5p-VSNL1 pathway; CDR1as-p53/ } \\
\text { MDM2 complex pathway }\end{array}$ & $(55,56)$ \\
\hline Melanoma & Promotes invasion in vitro and metastasis in vivo & Down & CDR1as-IGF2BP3 axis pathway & $(59,60)$ \\
\hline $\begin{array}{l}\text { Bladder } \\
\text { cancer }\end{array}$ & $\begin{array}{l}\text { Inhibiting cell proliferation, invasion and migration; induced the } \\
\text { apoptosis and enhanced the cisplatin chemosensitivity }\end{array}$ & Down & $\begin{array}{l}\text { CDR1as-miR-135a-p21 pathway; CDR1as-miR-1270/ } \\
\text { APAF1 axis pathway }\end{array}$ & $(61,62)$ \\
\hline GC & $\begin{array}{l}\text { An independent risk factor of overall survival; Correlates with a } \\
\text { more aggressive oncogenic phenotype }\end{array}$ & Up & CDR1as-miR-7-PTEN/PI3K/AKT pathway & (63) \\
\hline Osteosarcoma & $\begin{array}{l}\text { Impairing cell vitality and increased apoptosis and G1/S arrest in } \\
\text { parallel with reduced ability of cell migration }\end{array}$ & Up & CDR1as-miR-7-EGFR/CCNE1/PI3K/RAF1pathway & (64) \\
\hline
\end{tabular}

HCC hepatocellular carcinoma CCA, cholangiocarcinoma; NSCLC, non-small cell lung cancer; CRC, colorectal cancer; ESCC, esophageal squamous cell carcinoma; GC, gastric cancer.

(MVI) and promotes the proliferation and invasion of HCC cells by inhibiting the expression of mir-7 and its downstream target genes CCNE1, PIK3CD, KLF4, and p70S6K (Figure 1A). Jiang et al. evaluated the relationship between CDR1as expression levels and clinicopathological features of cholangiocarcinoma (CCA) and assessed the association of CDR1as expression with
OS. The results showed that upregulation of CDR1as was closely associated with advanced TNM stage, lymph node invasion, and postoperative recurrence, and the OS of patients with CCA with high CDRlas expression was worse than that of patients with CCA with low CDRlas expression (48). CDR1as binds to Mir641 and accelerates the degradation of Mir-641, which may lead

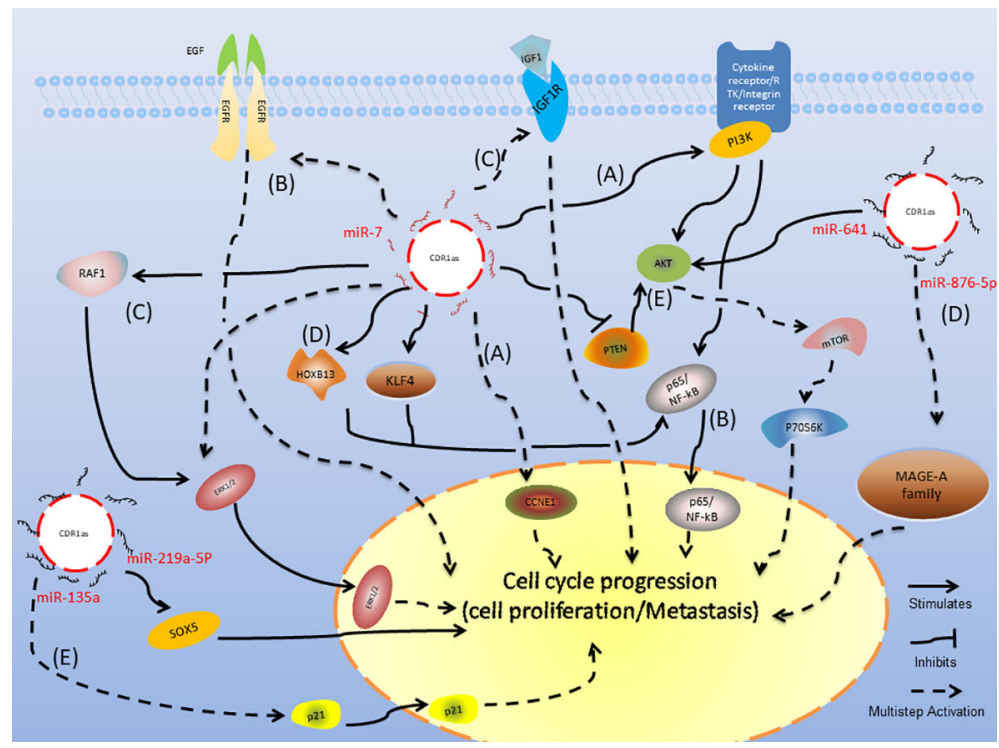

FIGURE 1 | Mechanism of CDR1as in various cancers. (A-C) CDR1as regulates the signaling pathways of HCC, NSCLC, and CRC processes. (D) CDR1as regulates the signaling pathways of ESCC processes. (E) CDR1as regulates the signaling pathways of other cancers. 
to upregulation of relative mRNA levels of $A K T 3$ and $m$ TOR and thus promote tumor progression (49). These data indicate that CDR1as plays an important regulatory role in the progression of HCC and may provide insights for the treatment of HCC and CCA.

\section{CDR1as in Non-Small Cell Lung Cancer}

In non-small cell lung cancer tissues, the expression of CDR1as is upregulated compared with para-carcinoma tissues. The dysregulation of CDR1as is significantly related to the clinical features and prognosis of NSCLC (44). Su et al. (45) confirmed that upregulation of CDR1as could inhibit the expression of $m i R$ 7 and that it correlates with advanced histopathological grade, larger tumor size, and more severe lymph node metastasis. Furthermore, researchers have confirmed that overexpression of CDR1as and downregulation of mir-7 increases the proliferation, metastasis, and invasion ability of NSCLC cells by the CDR1as/miR-7/NF-kB (p65) axis (Figure 1B). Zhang et al. demonstrated that patients with high expression of CDR1as had a high TNM stage, more frequent lymph node metastasis, shorter OS time, and that CDR1as was an independent prognostic factor for patients with NSCLC. Mechanistically, CDR1as functioned as a miR-7 sponge to upregulate the key target genes of $m i R-7$, including EGFR, CCNE1, and PIK3CD (46) (Figures 1A, B). Li et al. indicated that knockdown of circCDRlas inhibited the progression of NSCLC by decreasing cell viability, migration, and invasion, and increasing apoptosis by upregulating $m i R-219 a-5 p$ and downregulating SOX5 (47). These studies indicate that CDRlas may play an important role in the development of NSCLC and may contribute to the diagnosis and prognosis of NSCLC.

\section{CDR1as in Colorectal Cancer}

Tang et al. found that the expression of CDR1as was upregulated in CRC tissues compared to the adjacent normal mucosa. High expression of CDR1as was positively correlated with tumor size, T stage, lymph node metastasis, and poor OS. Dysregulation of CDR1as inhibited CRC cell proliferation and suppressed EGFR and $I G F-1 R$ expression by blocking miR-7expression (50) (Figures 1B, C). Weng et al. analyzed the clinical significance of CDRlas in CRC patients and found that CDRlas was significantly upregulated in CRC tissues compared with matched normal mucosae. Furthermore, they revealed by multivariate survival analysis that CDR1as could be an independent risk factor for OS. Upregulation of CDR1as led to blocking of $m i R-7$ and resulted in a more aggressive oncogenic phenotype, and subsequent activation of EGFR and RAF1 oncogenes in HCT116 and HT29 cells (51) (Figure 1C). These findings suggest that CDR1as could be a prognostic biomarker in CRC patients and may serve as a therapeutic target in CRC patients.

\section{CDR1as in Esophageal Squamous Cell Carcinoma}

A study performed by Sang et al. suggested that CDRlas is upregulated in ESCC and that it is associated with poor clinicopathological parameters in ESCC patients. High expression of ciRS-7 increased the proliferation, migration, and invasion abilities of ESCC cells. Mechanistic studies revealed that CDRlas acts as a sponge for miR-876-5p in ESCC cells. Furthermore, CDR1as reverses miR-876-5p-mediated repression of the tumor antigen MAGE-A family in ESCC cells (52) (Figure 1D). Li et al. (53) evaluated the expression of CDRlas in 123 ESCC patients and found that CDRlas expression was significantly upregulated in ESCC tissues compared with normal tissues. High expression of CDR1as was positively correlated with poor survival and advanced TNM stage, and inhibited the tumor inhibition of miR-7, including cell proliferation, migration, and invasion. Research on the mechanisms revealed that CDR1as functioned as a sponge for miR-7 and reactivated its downstream HOXB13-mediated NF$\kappa B$ pathway (Figure 1D). Huang et al. demonstrated that the expression of ciRS-7 was significantly increased in ESCC tissues and cells compared with their corresponding controls, and that ciRS-7 triggers the migration and invasion of ESCC via miR-7/ KLF4 and NF- $\mathrm{BB}$ signals (54). These studies indicate that CDRlas may play an important role in the development of ESCC and might contribute to the diagnosis and prognosis of ESCC.

\section{CDR1as in Brain Tumor}

CDRlas was identified as a circRNA in the brain and it is highly expressed in normal brain tissues (65). Barbagallo et al. observed that the expression of CDR1as is significantly decreased in glioblastoma multiforme biopsies and compared to normal brain parenchyma (average change $=-3.51$-fold), in glioblastoma multiforme cell lines and other cancer cell lines, except HCT116. Furthermore, the researchers identified CDR1as as a downstream miR-671-5p target in glioblastoma multiforme and that it is the only circRNA known to be targeted and degraded by miR-671-5p $(55,65)$. Lou et al. revealed that CDRlas expression decreased with the increase in glioma grade and that it was a reliable independent predictor of overall glioma survival. In terms of mechanism, CDR1as functions as a tumor suppressor by binding directly to $p 53$ at its DBD region to restrict MDM2 interaction (56).

\section{CDR1as in Head and Neck Cancer}

Zhang et al. collected 30 laryngeal squamous cell carcinoma (LSCC) tissues and corresponding relative normal tissues to determine the expression of CDRlas and their clinical significance. The results indicated that patients with high TNM stages, poorly differentiated tumors, lymph node metastases, and poor prognosis had high CDR1as levels. Overexpression of CDRlas enhanced cell vitality and promoted the proliferation, migration, and invasion of two LSCC cell lines. Mechanistically, CDRlas functioned as miR-7 sponges and upregulated the key targets of miR-7, CCNE1, and PIK3CD (57). Zhong et al. examined 44 nasopharyngeal carcinoma (NPC) tissues and 20 non-tumor tissues, and the results confirmed that CDR1as was highly expressed in NPC tissues and cell lines, which might be related to the promotion of NPC cells development. Moreover, CDR1as could upregulate E2F3 expression by binding to miR-7$5 \mathrm{p}$, and promote the growth and glucose metabolism of NPC 
cells (58). These results indicate that CDR1as is an oncogene in NPC and LSCC.

\section{CDR1as in Melanoma}

Zhang et al. predicted the regulatory role of CDR1as in melanoma through bioinformatics analysis and showed that CDRlas may act as a competing endogenous RNA for the vital genes, which are associated with the invasion and migration of melanoma (59). Recent research has revealed that epigenetic silencing of LINC00632 can lead to the depletion of CDR1as and promote invasion in vitro, and metastasis in vivo through a $m i R$ 7-independent, IGF2BP3-mediated mechanism (60). These results indicate that $\mathrm{CDR} 1$ as plays an important regulatory role in the biological process of melanoma.

\section{CDR1as in Bladder Cancer}

$\mathrm{Li}$ et al. found that the expression of CDRlas in bladder cancer was significantly lower than that in adjacent tissues. CDR1as overexpression inhibits the proliferation, invasion, and migration of bladder cancer cells and directly binds to miR-135a and inhibits its activity in bladder cancer (61) (Figure 1E). Yuan et al. reported that Cdr1as induced apoptosis and enhanced the chemosensitivity of bladder cancer cells both in vitro and in vivo. We verified that CDRlas exerts a cisplatin-chemosensitization effect on bladder cancer cells through the CDR1as/miR-1270/APAF1 axis (62).

\section{CDR1as in Other Cancers}

A study by Pan et al. investigated the clinical significance of CDRlas in 102 primary gastric cancer tissues and matched paracarcinoma tissues, and subsequently confirmed the clinical relevance in an independent validation cohort. The results indicated that CDR1as expression was higher in gastric cancer tissues than in para-carcinoma tissues, and increased with the tumor stage. The overexpression of CDRlas blocks tumor inhibition induced by miR-7 in MGC-803 and HGC-27 cells by antagonizing the miR-7-mediated PTEN/PI3K/AKT pathway (Figure 1E), leading to a more aggressive oncogenic phenotype (63).

Uhr et al. revealed the relationship between miR-7 and its binding compound CDR1as and the prognosis and prediction of first-line tamoxifen treatment for breast cancer (66). Xu et al. showed that CDR1as was upregulated in osteosarcoma tissues and its expression was positively correlated with tumor size and distant metastasis. Knockdown of CDR1as leads to de-repression of miR-7 levels and inhibition of its target genes, including EGFR, CCNE1, PI3KCD, and RAF1, impaired cell vitality and increased apoptosis and G1/S arrest in parallel with reduced cell migration ability (64). In addition, CDRlas has been reported to play an important role in the development of ovarian cancer and pancreatic cancer $(67,68)$.

\section{CDR1as as a Biomarker in Cancer}

An increasing number of studies have shown that upregulation of CDR1as is associated with prognosis in various types of cancer $(69,70)$. We performed a comprehensive literature search from a public database (PubMed, Medline, and Web of Science) to identify all eligible studies describing the use of CDRlas as a prognostic factor for different types of cancer. A total of eight studies were included in this study $(45,46,48,50,51,53,57,63)$. The results showed that high expression of CDR1as was significantly associated with poor OS ( $\mathrm{HR}=2.50,95 \% \mathrm{CI}: 2.06$ -3.04; $p<0.001$ ) (Figure 2). Furthermore, the results revealed the prognostic significance of CDR1as in digestive system neoplasms $(\mathrm{HR}=1.69,95 \% \mathrm{CI}=2.14-2.71 ; p<0.001)$, CRC $(\mathrm{HR}=1.34,95 \% \mathrm{CI}=1.96-2.85 ; p<0.001)$, and NSCLC $(\mathrm{HR}=$ $2.40,95 \% \mathrm{CI}=3.42-4.83 ; p=0.008)$ (Figure 3). High expression levels of CDRlas were also associated with TNM stage $(\mathrm{OR}=$ $2.13,95 \% \mathrm{CI}=1.63-2.78 ; p=0.001)$, distant metastases $(\mathrm{OR}=$ $3.50,95 \% \mathrm{CI}=1.90-6.64 ; p=0.008)$, and lymph node metastases $(\mathrm{OR}=1.68,95 \% \mathrm{CI}=1.24-2.26 ; p=0.001)$, but not with the differentiation status and lymph node invasion (Figure 4). These results suggest that the expression of CDRlas may be an effective biomarker for the diagnosis, treatment, and prognosis of tumors.

\section{CONCLUSIONS}

At present, circRNA has become a new field in disease research, including diseases such as autoimmune (71), gynecological (72, 73), respiratory system (74), and cancer (75-78). CircRNAs not only play an important role in tumor regulation, but can also be used as biological markers for tumor diagnosis and treatment. It has been pointed out that Hsa_ circ_0000190 has a low expression level in gastric cancer plasma and tissues, and its sensitivity and specificity as a diagnostic marker are even better than CEA and CA19-9 (79). Hsa_circ_0005075 has high specificity and sensitivity for the diagnosis and treatment of HCC (80). Therefore, the expression and characteristics of circRNAs can be used as biological markers of tumors.

As a well-known circRNA, CDR1as plays an important role in the progression of many diseases. In recent years, more studies on the pathogenesis, progression, and mechanism of CDRlas have been conducted. Differences in the expression of CDR1as in various tumors are obvious. CDRlas regulates the biological behavior of tumor cells in many aspects, including cell proliferation, differentiation, metastasis, and apoptosis. CDR1as mainly regulates gene expression at the transcriptional and posttranscriptional levels. It is a strictly controlled biological process, rather than a random splicing error. Its main form of action in cancer is as a miRNA sponge, which makes the competing endogenous RNA network more complex. Furthermore, we integrated the prognostic information of CDRlas in different tumors, and found that the higher the expression of CDR1as, the shorter the survival time, and the worse the prognosis, especially in patients with digestive system tumors. In addition, the evaluation of TNM stage, distant metastasis, and lymph node metastasis in tumor patients are of great value.

In this study, we reviewed the characteristics, biological functions, and clinical application value of CDR1as in detail, and mainly examined its expression in various tumors, its influence on prognosis of tumor patients, and its role in tumor 
Study

ID
HR (95\% Cl)

Weight
Su 2017

Zhang 2018

Jiang 2017

Tang 2017

Weng 2017(Training cohort)

Weng 2018(Validation cohort)

Li 2018

Pan 2018 (testing cohort:

Pan 2018 (validation cohort)

Zhang 2018

Overall $(1-$ squared $=54.3 \%, p=0.020)$

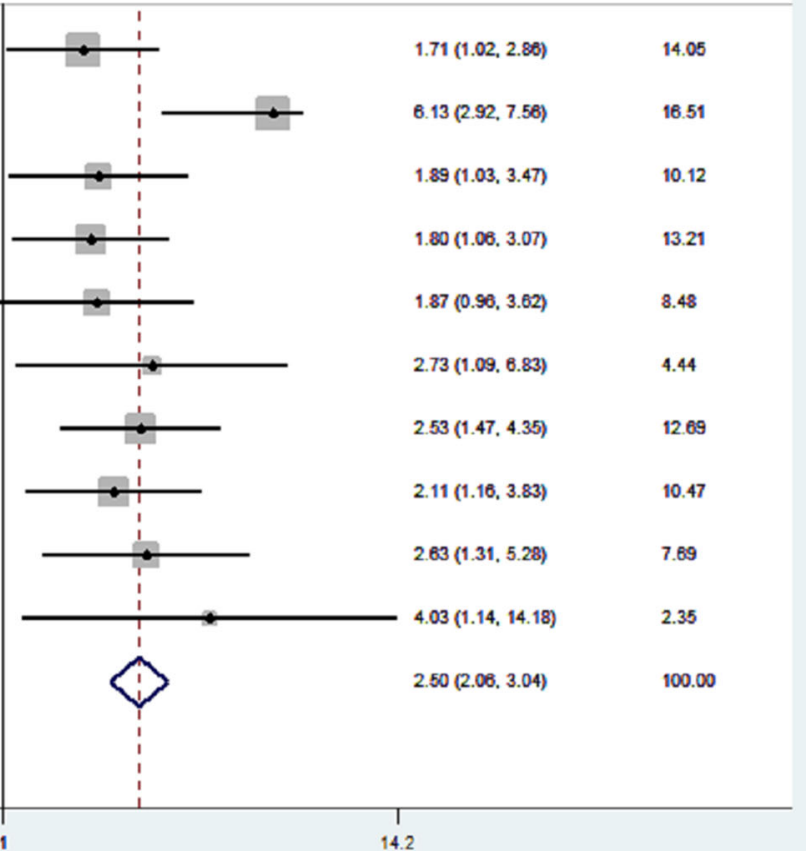

14.2

FIGURE 2 | Forest plot of the hazard ratio (HR) for CDR1as increase and overall survival.

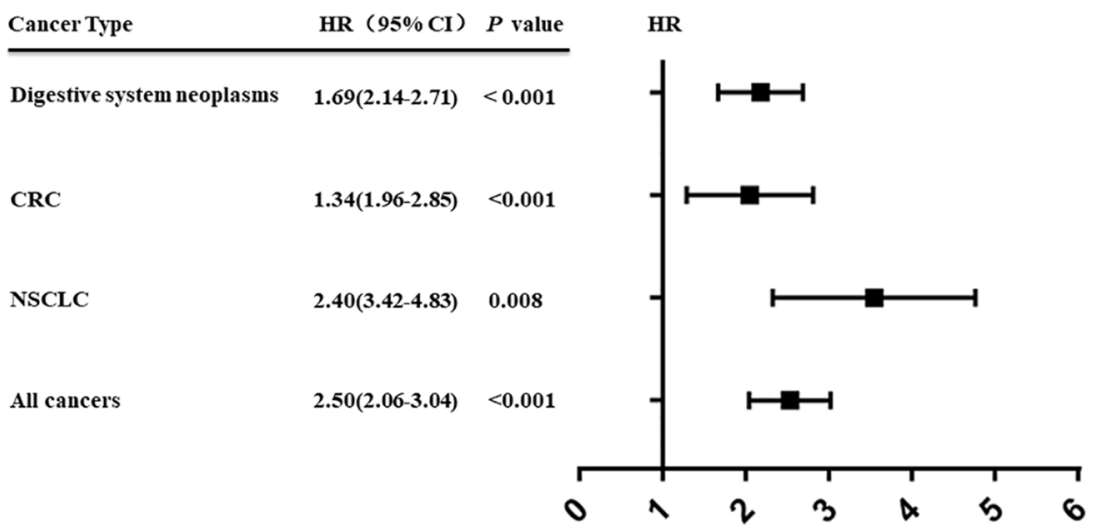

FIGURE 3 | High expression of CDR1as indicates poor prognosis of tumor.

signaling pathways. The high expression of CDRlas in tumors promotes the ability of tumor cells to differentiate, proliferate and metastasize, leading to the poor prognosis of cancer patients. Although the current research on CDR1as is not very thorough, and its detailed mechanism for regulating tumor progression is not very clear, CDR1as plays an irreplaceable role in the process of tumorigenesis and may become a potential target for cancer prevention and treatment. 


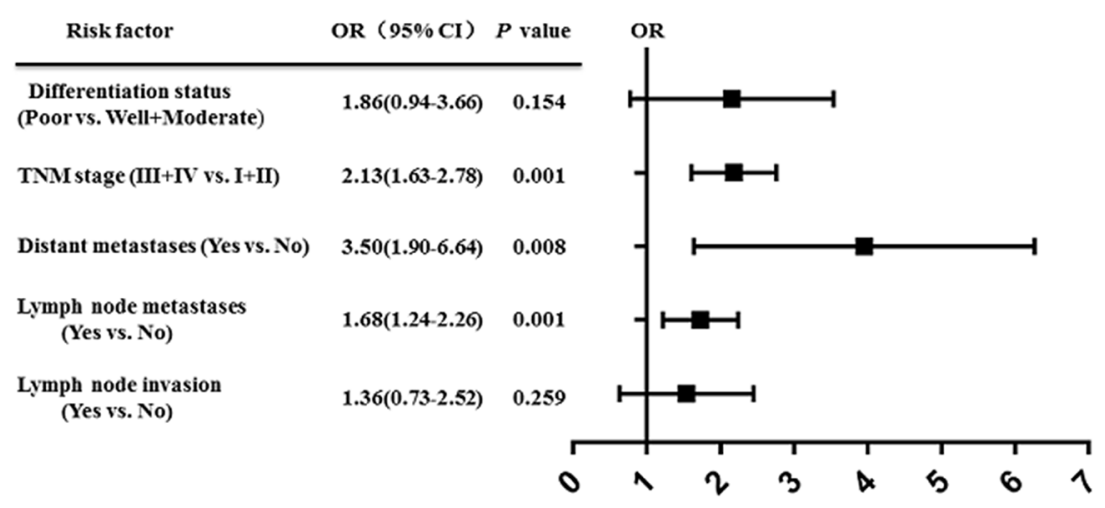

FIGURE 4 | High expression of CDR1as is related to the clinicopathology of tumor.

\section{DATA AVAILABILITY STATEMENT}

The datasets presented in this study can be found in online repositories. The names of the repository/repositories and accession number(s);, can be found in the article/supplementary material.

\section{AUTHOR CONTRIBUTIONS}

All authors have contributed to the preparation of this manuscript. FJ, RY, XW, and XJ were responsible for collecting the data, analysis, and drafting the first copy. YP and LN were

\section{REFERENCES}

1. Siegel RL, Miller KD, Jemal A. Cancer statistics, 2017. CA Cancer J Clin (2017) 67:7-30. doi: 10.3322/caac.21387

2. Cui X, Wang J, Guo Z, Li M, Li M, Liu S, et al. Emerging function and potential diagnostic value of circular rnas in cancer. Mol Cancer (2018) 17:123. doi: 10.1186/s12943-018-0877-y

3. Qiu M, Xia W, Chen R, Wang S, Xu Y, Ma Z, et al. The circular rna circprkci promotes tumor growth in lung adenocarcinoma. Cancer Res (2018) 78:283951. doi: 10.1158/0008-5472.CAN-17-2808

4. Sanger HL, Klotz G, Riesner D, Gross HJ, Kleinschmidt AK. Viroids are single-stranded covalently closed circular rna molecules existing as highly base-paired rod-like structures. Proc Natl Acad Sci U S A (1976) 73:3852-6. doi: $10.1073 /$ pnas.73.11.3852

5. Hsu MT, Coca-Prados M. Electron microscopic evidence for the circular form of rna in the cytoplasm of eukaryotic cells. Nature (1979) 280:339-40. doi: $10.1038 / 280339 \mathrm{a} 0$

6. Salzman J, Gawad C, Wang PL, Lacayo N, Brown PO. Circular rnas are the predominant transcript isoform from hundreds of human genes in diverse cell types. PLoS One (2012) 7:e30733. doi: 10.1371/journal.pone.0030733

7. Barrett SP, Salzman J. Circular rnas: Analysis, expression and potential functions. Development (2016) 143:1838-47. doi: 10.1242/dev.128074

8. Memczak S, Jens M, Elefsinioti A, Torti F, Krueger J, Rybak A, et al. Circular rnas are a large class of animal rnas with regulatory potency. Nature (2013) 495:333-8. doi: 10.1038/nature11928

9. Bachmayr-Heyda A, Reiner AT, Auer K, Sukhbaatar N, Aust S, BachleitnerHofmann T, et al. Correlation of circular rna abundance with proliferationexemplified with colorectal and ovarian cancer, idiopathic lung fibrosis, and normal human tissues. Sci Rep (2015) 5:8057. doi: 10.1038/srep08057 responsible for editing the manuscript. $\mathrm{PZ}, \mathrm{NG}$, and $\mathrm{BM}$ were responsible for the theme, final editing, and preparation of the manuscript for submission. All authors contributed to the article and approved the submitted version.

\section{FUNDING}

This study was supported by the Natural Science Foundation of Anhui Province (grant number 1808085 MH273) and the first Affiliated Hospital of Anhui Medical University National Natural Science Foundation Cultivation fund (grant number 2018kj24).
10. Burd CE, Jeck WR, Liu Y, Sanoff HK, Wang Z, Sharpless NE. Expression of linear and novel circular forms of an ink4/arf-associated non-coding rna correlates with atherosclerosis risk. PLoS Genet (2010) 6:e1001233. doi: 10.1371/journal.pgen.1001233

11. Lukiw WJ. Circular rna (circrna) in alzheimer's disease (ad). Front Genet (2013) 4:307. doi: 10.3389/fgene.2013.00307

12. Li P, Chen S, Chen H, Mo X, Li T, Shao Y, et al. Using circular rna as a novel type of biomarker in the screening of gastric cancer. Clin Chim Acta (2015) 444:132-6. doi: 10.1016/j.cca.2015.02.018

13. Bolha L, Ravnik-Glavac M, Glavac D. Circular rnas: Biogenesis, function, and a role as possible cancer biomarkers. Int J Genomics (2017) 2017:6218353. doi: $10.1155 / 2017 / 6218353$

14. Sheng JQ, Liu L, Wang MR, Li PY. Circular rnas in digestive system cancer: Potential biomarkers and therapeutic targets. Am J Cancer Res (2018) 8:114256.

15. Geng Y, Jiang J, Wu C. Function and clinical significance of circrnas in solid tumors. J Hematol Oncol (2018) 11:98. doi: 10.1186/s13045-018-0643-Z

16. Sang Y, Chen B, Song X, Li Y, Liang Y, Han D, et al. Circrna_0025202 regulates tamoxifen sensitivity and tumor progression via regulating the mir182-5p/foxo3a axis in breast cancer. Mol Ther (2019) 27:1638-52. doi: 10.1016/j.ymthe.2019.05.011

17. Zeng $\mathrm{K}$, Chen $\mathrm{X}, \mathrm{Xu} \mathrm{M}$, Liu $\mathrm{X}, \mathrm{Hu} \mathrm{X}, \mathrm{Xu} \mathrm{T}$, et al. Circhipk3 promotes colorectal cancer growth and metastasis by sponging mir-7. Cell Death Dis (2018) 9:417. doi: 10.1038/s41419-018-0454-8

18. Liu F, Zhang H, Xie F, Tao D, Xiao X, Huang C, et al. Hsa_circ_0001361 promotes bladder cancer invasion and metastasis through mir-491-5p/mmp9 axis. Oncogene (2020) 39:1696-709. doi: 10.1038/s41388-019-1092-z

19. Xia W, Qiu M, Chen R, Wang S, Leng X, Wang J, et al. Circular rna has_circ_0067934 is upregulated in esophageal squamous cell carcinoma and promoted proliferation. Sci Rep (2016) 6:35576. doi: 10.1038/srep35576 
20. Han D, Li J, Wang H, Su X, Hou J, Gu Y, et al. Circular rna circmtol acts as the sponge of microrna-9 to suppress hepatocellular carcinoma progression. Hepatology (2017) 66:1151-64. doi: 10.1002/hep.29270

21. Guo W, Zhang J, Zhang D, Cao S, Li G, Zhang S, et al. Polymorphisms and expression pattern of circular rna circ-itch contributes to the carcinogenesis of hepatocellular carcinoma. Oncotarget (2017) 8:48169-77. doi: 10.18632/ oncotarget. 18327

22. Yu J, Xu QG, Wang ZG, Yang Y, Zhang L, Ma JZ, et al. Circular rna csmarca5 inhibits growth and metastasis in hepatocellular carcinoma. J Hepatol (2018) 68:1214-27. doi: 10.1016/j.jhep.2018.01.012

23. Huang XY, Huang ZL, Xu YH, Zheng Q, Chen Z, Song W, et al. Comprehensive circular rna profiling reveals the regulatory role of the circrna-100338/mir-141-3p pathway in hepatitis b-related hepatocellular carcinoma. Sci Rep (2017) 7:5428. doi: 10.1038/s41598-017-05432-8

24. Li WH, Song YC, Zhang H, Zhou ZJ, Xie X, Zeng QN, et al. Decreased expression of hsa_circ_00001649 in gastric cancer and its clinical significance. Dis Markers (2017) 2017:4587698. doi: 10.1155/2017/4587698

25. Ji W, Qiu C, Wang M, Mao N, Wu S, Dai Y. Hsa_circ_0001649: A circular rna and potential novel biomarker for colorectal cancer. Biochem Biophys Res Commun (2018) 497:122-26. doi: 10.1016/j.bbrc.2018.02.036

26. Qin M, Liu G, Huo X, Tao X, Sun X, Ge Z, et al. Hsa_circ_0001649: A circular rna and potential novel biomarker for hepatocellular carcinoma. Cancer Biomark (2016) 16:161-9. doi: 10.3233/CBM-150552

27. Xu Y, Yao Y, Zhong X, Leng K, Qin W, Qu L, et al. Downregulated circular rna hsa_circ_0001649 regulates proliferation, migration and invasion in cholangiocarcinoma cells. Biochem Biophys Res Commun (2018) 496:45561. doi: 10.1016/j.bbrc.2018.01.077

28. Guarnerio J, Bezzi M, Jeong JC, Paffenholz SV, Berry K, Naldini MM, et al. Oncogenic role of fusion-circrnas derived from cancer-associated chromosomal translocations. Cell (2016) 165:289-302. doi: 10.1016/ j.cell.2016.03.020

29. Szabo L, Morey R, Palpant NJ, Wang PL, Afari N, Jiang C, et al. Statistically based splicing detection reveals neural enrichment and tissue-specific induction of circular rna during human fetal development. Genome Biol (2015) 16:126. doi: 10.1186/s13059-015-0690-5

30. Hansen TB, Kjems J, Damgaard CK. Circular rna and mir-7 in cancer. Cancer Res (2013) 73:5609-12. doi: 10.1158/0008-5472.CAN-13-1568

31. Hansen TB, Jensen TII, Clausen BH, Bramsen JB, Finsen B, Damgaard CK, et al. Natural rna circles function as efficient microrna sponges. Nature (2013) 495:384-8. doi: 10.1038/nature11993

32. Qiu LP, Wu YH, Yu XF, Tang Q, Chen L, Chen KP. The emerging role of circular rnas in hepatocellular carcinoma. J Cancer (2018) 9:1548-59. doi: $10.7150 /$ jca. 24566

33. Peng L, Yuan XQ, Li GC. The emerging landscape of circular rna cirs-7 in cancer (review). Oncol Rep (2015) 33:2669-74. doi: 10.3892/or.2015.3904

34. Akhter R. Circular rna and alzheimer's disease. Adv Exp Med Biol (2018) 1087:239-43. doi: 10.1007/978-981-13-1426-1_19

35. Zhang Y, Sun L, Xuan L, Pan Z, Li K, Liu S, et al. Reciprocal changes of circulating long non-coding rnas zfas 1 and cdrlas predict acute myocardial infarction. Sci Rep (2016) 6:22384. doi: 10.1038/srep22384

36. Xu H, Guo S, Li W, Yu P. The circular rna cdrlas, via mir-7 and its targets, regulates insulin transcription and secretion in islet cells. Sci Rep (2015) 5:12453. doi: 10.1038/srep12453

37. Kristensen LS, Hansen TB, Veno MT, Kjems J. Circular rnas in cancer: Opportunities and challenges in the field. Oncogene (2018) 37:555-65. doi: 10.1038/onc.2017.361

38. Meng S, Zhou H, Feng Z, Xu Z, Tang Y, Li P, et al. Circrna: Functions and properties of a novel potential biomarker for cancer. Mol Cancer (2017) 16:94. doi: 10.1186/s12943-017-0663-2

39. Dong Y, He D, Peng Z, Peng W, Shi W, Wang J, et al. Circular rnas in cancer: An emerging key player. J Hematol Oncol (2017) 10:2. doi: 10.1186/s13045016-0370-2

40. Yu L, Gong X, Sun L, Zhou Q, Lu B, Zhu L. The circular rna cdrlas act as an oncogene in hepatocellular carcinoma through targeting mir-7 expression. PLoS One (2016) 11:e0158347. doi: 10.1371/journal.pone.0158347

41. Yang X, Xiong Q, Wu Y, Li S, Ge F. Quantitative proteomics reveals the regulatory networks of circular rna cdrlas in hepatocellular carcinoma cells. J Proteome Res (2017) 16:3891-902. doi: 10.1021/acs.jproteome.7b00519
42. Xu L, Zhang M, Zheng X, Yi P, Lan C, Xu M. The circular rna cirs-7 (cdrlas) acts as a risk factor of hepatic microvascular invasion in hepatocellular carcinoma. J Cancer Res Clin Oncol (2017) 143:17-27. doi: 10.1007/s00432016-2256-7

43. Chen L, Shi J, Wu Y, Qiu R, Zeng L, Lou L, et al. Circrna cdrlas promotes hepatoblastoma proliferation and stemness by acting as a mir-7-5p sponge to upregulate klf4 expression. Aging (Albany NY) (2020) 12:19233-53. doi: 10.18632/aging.103748

44. Yan B, Zhang W, Mao XW, Jiang LY. Circular rna cirs-7 correlates with advance disease and poor prognosis, and its down-regulation inhibits cells proliferation while induces cells apoptosis in non-small cell lung cancer. Eur Rev Med Pharmacol Sci (2018) 22:8712-21. doi: 10.26355/eurrev_201812 16636

45. Su C, Han Y, Zhang H, Li Y, Yi L, Wang X, et al. Cirs-7 targeting mir-7 modulates the progression of non-small cell lung cancer in a manner dependent on nf-kappab signalling. J Cell Mol Med (2018) 22:3097-107. doi: $10.1111 / \mathrm{jcmm} .13587$

46. Zhang X, Yang D, Wei Y. Overexpressed cdrlas functions as an oncogene to promote the tumor progression via mir-7 in non-small-cell lung cancer. Onco Targets Ther (2018) 11:3979-87. doi: 10.2147/OTT.S158316

47. Li Y, Zhang J, Pan S, Zhou J, Diao X, Liu S. Circrna cdrlas knockdown inhibits progression of non-small-cell lung cancer by regulating mir-219a-5p/sox 5 axis. Thorac Cancer (2020) 11:537-48. doi: 10.1111/1759-7714.13274

48. Jiang XM, Li ZL, Li JL, Xu Y, Leng KM, Cui YF, et al. A novel prognostic biomarker for cholangiocarcinoma: Circrna cdrlas. Eur Rev Med Pharmacol Sci (2018) 22:365-71. doi: 10.26355/eurrev_201801_14182

49. Li D, Tang Z, Gao Z, Shen P, Liu Z, Dang X. Circular rna cdrlas exerts oncogenic properties partially through regulating microrna 641 in cholangiocarcinoma. Mol Cell Biol (2020) 40:e00042-20. doi: 10.1128/ MCB.00042-20

50. Tang W, Ji M, He G, Yang L, Niu Z, Jian M, et al. Silencing cdrlas inhibits colorectal cancer progression through regulating microrna-7. Onco Targets Ther (2017) 10:2045-56. doi: 10.2147/OTT.S131597

51. Weng W, Wei Q, Toden S, Yoshida K, Nagasaka T, Fujiwara T, et al. Circular rna cirs-7-a promising prognostic biomarker and a potential therapeutic target in colorectal cancer. Clin Cancer Res (2017) 23:3918-28. doi: 10.1158/ 1078-0432.CCR-16-2541

52. Sang M, Meng L, Sang Y, Liu S, Ding P, Ju Y, et al. Circular rna cirs-7 accelerates escc progression through acting as a mir-876-5p sponge to enhance mage-a family expression. Cancer Lett (2018) 426:37-46. doi: 10.1016/j.canlet.2018.03.049

53. Li RC, Ke S, Meng FK, Lu J, Zou XJ, He ZG, et al. Cirs-7 promotes growth and metastasis of esophageal squamous cell carcinoma via regulation of mir-7/ hoxb13. Cell Death Dis (2018) 9:838. doi: 10.1038/s41419-018-0852-y

54. Huang H, Wei L, Qin T, Yang N, Li Z, Xu Z. Circular rna cirs-7 triggers the migration and invasion of esophageal squamous cell carcinoma via mir-7/klf4 and nf-kappab signals. Cancer Biol Ther (2019) 20:73-80. doi: 10.1080/ 15384047.2018.1507254

55. Barbagallo D, Condorelli A, Ragusa M, Salito L, Sammito M, Banelli B, et al. Dysregulated mir-671-5p / cdrl-as / cdr1 / vsnl1 axis is involved in glioblastoma multiforme. Oncotarget (2016) 7:4746-59. doi: 10.18632/ oncotarget.6621

56. Lou J, Hao Y, Lin K, Lyu Y, Chen M, Wang H, et al. Circular rna cdrlas disrupts the p53/mdm2 complex to inhibit gliomagenesis. Mol Cancer (2020) 19:138. doi: 10.1186/s12943-020-01253-y

57. Zhang J, Hu H, Zhao Y, Zhao Y. Cdrlas is overexpressed in laryngeal squamous cell carcinoma to promote the tumour's progression via mir-7 signals. Cell Prolif (2018) 51:e12521. doi: 10.1111/cpr.12521

58. Zhong Q, Huang J, Wei J, Wu R. Circular rna cdrlas sponges mir-7-5p to enhance e2f3 stability and promote the growth of nasopharyngeal carcinoma. Cancer Cell Int (2019) 19:252. doi: 10.1186/s12935-019-0959-y

59. Zhang L, Li Y, Liu W, Li H, Zhu Z. Analysis of the complex interaction of cdrlas-mirna-protein and detection of its novel role in melanoma. Oncol Lett (2018) 16:1219-25. doi: 10.3892/ol.2018.8700

60. Hanniford D, Ulloa-Morales A, Karz A, Berzoti-Coelho MG, Moubarak RS, Sanchez-Sendra B, et al. Epigenetic silencing of cdrlas drives igf $2 \mathrm{bp} 3$ mediated melanoma invasion and metastasis. Cancer Cell (2020) 37:55-70 e15. doi: 10.1016/j.ccell.2019.12.007 
61. Li P, Yang X, Yuan W, Yang C, Zhang X, Han J, et al. Circrna-cdrlas exerts anti-oncogenic functions in bladder cancer by sponging microrna-135a. Cell Physiol Biochem (2018) 46:1606-16. doi: 10.1159/000489208

62. Yuan W, Zhou R, Wang J, Han J, Yang X, Yu H, et al. Circular rna cdrlas sensitizes bladder cancer to cisplatin by upregulating apafl expression through mir-1270 inhibition. Mol Oncol (2019) 13:1559-76. doi: 10.1002/1878-0261.12523

63. Pan H, Li T, Jiang Y, Pan C, Ding Y, Huang Z, et al. Overexpression of circular rna cirs-7 abrogates the tumor suppressive effect of mir-7 on gastric cancer via pten/pi3k/akt signaling pathway. J Cell Biochem (2018) 119:440-46. doi: $10.1002 /$ jcb. 26201

64. Xu B, Yang T, Wang Z, Zhang Y, Liu S, Shen M. Circrna cdr1as/mir-7 signals promote tumor growth of osteosarcoma with a potential therapeutic and diagnostic value. Cancer Manag Res (2018) 10:4871-80. doi: 10.2147/ CMAR.S178213

65. Hansen TB, Wiklund ED, Bramsen JB, Villadsen SB, Statham AL, Clark SJ, et al. Mirna-dependent gene silencing involving ago2-mediated cleavage of a circular antisense rna. EMBO J (2011) 30:4414-22. doi: 10.1038/emboj.2011.359

66. Uhr K, Sieuwerts AM, de Weerd V, Smid M, Hammerl D, Foekens JA, et al. Association of microrna-7 and its binding partner cdr1-as with the prognosis and prediction of 1(st)-line tamoxifen therapy in breast cancer. Sci Rep (2018) 8:9657. doi: 10.1038/s41598-018-27987-w

67. Chen H, Mao M, Jiang J, Zhu D, Li P. Circular rna cdrlas acts as a sponge of mir-135b-5p to suppress ovarian cancer progression. Onco Targets Ther (2019) 12:3869-79. doi: 10.2147/OTT.S207938

68. Liu L, Liu FB, Huang M, Xie K, Xie QS, Liu CH, et al. Circular rna cirs-7 promotes the proliferation and metastasis of pancreatic cancer by regulating mir-7-mediated egfr/stat3 signaling pathway. Hepatobiliary Pancreat Dis Int (2019) 18:580-86. doi: 10.1016/j.hbpd.2019.03.003

69. Zhang M, Xin Y. Circular rnas: A new frontier for cancer diagnosis and therapy. J Hematol Oncol (2018) 11:21. doi: 10.1186/s13045-018-0569-5

70. Zhang HD, Jiang LH, Sun DW, Hou JC, Ji ZL. Circrna: A novel type of biomarker for cancer. Breast Cancer (2018) 25:1-7. doi: 10.1007/s12282-017-0793-9

71. Zurawska A, Mycko MP, Selmaj KW. Circular rnas as a novel layer of regulatory mechanism in multiple sclerosis. J Neuroimmunol (2019) 334:576971. doi: 10.1016/j.jneuroim.2019.576971

72. Huang J, Zhou Q, Li Y. Circular rnas in gynecological disease: Promising biomarkers and diagnostic targets. Biosci Rep (2019) 39:BSR20181641. doi: 10.1042/BSR20181641
73. Xu X, Jia SZ, Dai Y, Zhang JJ, Li X, Shi J, et al. The relationship of circular rnas with ovarian endometriosis. Reprod Sci (2018) 25:1292-300. doi: 10.1177/ 1933719118759439

74. Huang Z, Cao Y, Zhou M, Qi X, Fu B, Mou Y, et al. Hsa_circ_0005519 increases il-13/il-6 by regulating hsa-let-7a-5p in cd4(+) t cells to affect asthma. Clin Exp Allergy (2019) 49:1116-27. doi: 10.1111/cea.13445

75. Hsiao KY, Lin YC, Gupta SK, Chang N, Yen L, Sun HS, et al. Noncoding effects of circular rna ccdc66 promote colon cancer growth and metastasis. Cancer Res (2017) 77:2339-50. doi: 10.1158/0008-5472.CAN-16-1883

76. Legnini I, Di Timoteo G, Rossi F, Morlando M, Briganti F, Sthandier O, et al. Circ-znf609 is a circular rna that can be translated and functions in myogenesis. Mol Cell (2017) 66:22-37.e9. doi: 10.1016/j.molcel.2017.02.017

77. Du WW, Yang W, Liu E, Yang Z, Dhaliwal P, Yang BB. Foxo3 circular rna retards cell cycle progression via forming ternary complexes with p21 and cdk2. Nucleic Acids Res (2016) 44:2846-58. doi: 10.1093/nar/gkw027

78. Abdelmohsen K, Panda AC, Munk R, Grammatikakis I, Dudekula DB, De S, et al. Identification of hur target circular rnas uncovers suppression of pabpn1 translation by circpabpn1. RNA Biol (2017) 14:361-69. doi: 10.1080/ 15476286.2017.1279788

79. Chen S, Li T, Zhao Q, Xiao B, Guo J. Using circular rna hsa_circ_0000190 as a new biomarker in the diagnosis of gastric cancer. Clin Chim Acta (2017) 466:167-71. doi: 10.1016/j.cca.2017.01.025

80. Shang X, Li G, Liu H, Li T, Liu J, Zhao Q, et al. Comprehensive circular rna profiling reveals that hsa_circ_0005075, a new circular rna biomarker, is involved in hepatocellular crcinoma development. Med (Baltimore) (2016) 95: e3811. doi: 10.1097/MD.0000000000003811

Conflict of Interest: The authors declare that the research was conducted in the absence of any commercial or financial relationships that could be construed as a potential conflict of interest.

Copyright (c) 2021 Jian, Yangyang, Wei, Jiadan, Na, Peng, Maohong, Guoping and Zhaoji. This is an open-access article distributed under the terms of the Creative Commons Attribution License (CC BY). The use, distribution or reproduction in other forums is permitted, provided the original author(s) and the copyright owner(s) are credited and that the original publication in this journal is cited, in accordance with accepted academic practice. No use, distribution or reproduction is permitted which does not comply with these terms. 